\title{
Universal antiretroviral therapy for pregnant and breastfeeding HIV-infected women: towards the elimination of mother-to-child transmission of HIV-I in resource-limited settings? Renaud Becquet ${ }^{1,2,3}$
}

Address: ${ }^{1}$ INSERM, Unité 897, Centre de Recherche "Epidémiologie et Biostatistique", Bordeaux, France, ${ }^{2}$ Institut de Santé Publique Epidémiologie Développement (ISPED), Université Victor Segalen Bordeaux 2, Bordeaux, France and ${ }^{3}$ On behalf of the ANRS 12200 Study Group from Fifth Dominique Dormont International Conference. Mother-to-child transmitted viral diseases: from transmission to children care Paris, France. 26-28 March 2009

Published: 22 July 2009

Retrovirology 2009, 6(SuppI I):L7 doi:I0.II86/I742-4690-6-SI-L7

This abstract is available from: http://www.retrovirology.com/content/6/SI/L7

(c) 2009 Becquet; licensee BioMed Central Ltd.

Several unmet scientific needs that account for the partial failure of MTCT prevention efforts in resource-constrained settings, particularly in Africa. Single-dose and short-course antiretroviral (ARV) regimens are only partially effective and have failed to achieve wide coverage despite their apparent simplicity. More potent ARV combinations are restricted to pregnant women who need treatment for themselves but are also infrequently used. Furthermore, postnatal transmission via breastfeeding is a serious additional threat. Modifications of infant feeding practices aim to reduce breast-milk HIV transmission: replacement feeding is neither affordable nor safe for the majority of African women, and early breastfeeding cessation (e.g. prior to 6 months of life) requires substantial care and nutritional counselling to be practised safely.

We argue that the recent roll out of highly active ARV treatment (HAART) has changed the paradigm of prevention of MTCT. To date, postnatal ARV interventions that have been evaluated target either maternal ARV treatment to selected breastfeeding women, with good efficacy, or single-drug post-exposure prophylaxis for short periods of time to their neonates, with a partial efficacy and at the expense of acquisition of drug-related viral resistance. We hypothesize that a viable solution to eliminate paediatric AIDS lies in the universal provision of HAART to all HIVinfected women through pregnancy, delivery, and covering the entire breastfeeding period.

We suggest the active promotion of the universal maternal HAART approach as a way towards elimination of MTCT in resource-limited settings. We argue that HAART should be made available to all HIV-infected pregnant women in resource-limited settings, irrespective of their CD4 count or clinical stage, and even to those who present late in pregnancy. This universal ARV-based strategy will be accompanied by proper pharmacovigilance systems. It should consider the breastfeeding cessation around six months of age, which implies the need for a proper and systematic nutritional support. Continuing investigations will compare the safety, acceptability, feasibility and efficiency of various maternal HAART regimens for preventing MTCT throughout the entire exposure period in order to rank them according to the best risk-benefit balance. 